
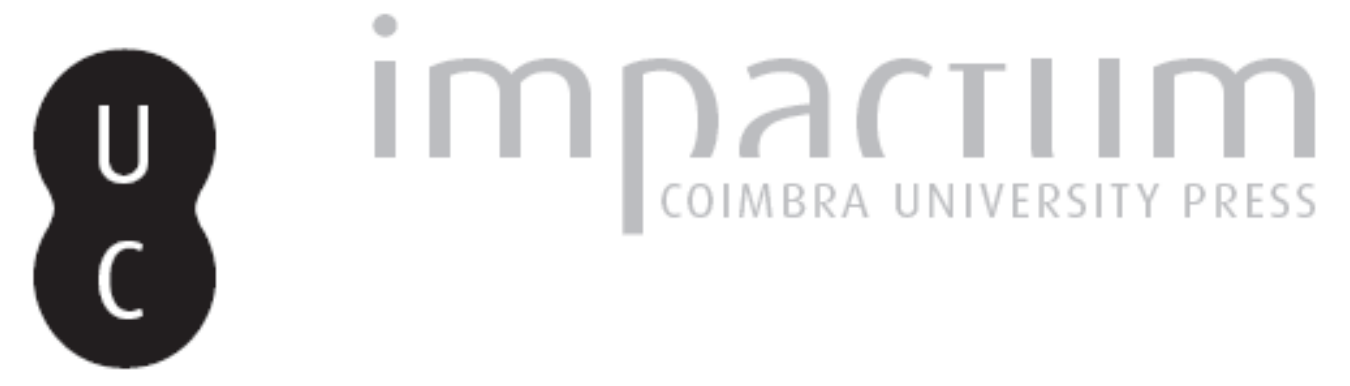

\title{
Paulmier de Gonneville et le Portugal: un navigateur normand dans la première mondialisation
}
Autor(es):
Maneuvrier, Christophe

Publicado por: Imprensa da Universidade de Coimbra

URL

persistente:

URI:http://hdl.handle.net/10316.2/40955

DOI:

DOI:https://doi.org/10.14195/1645-2259_16_5

Accessed : $\quad$ 26-Apr-2023 12:32:29

A navegação consulta e descarregamento dos títulos inseridos nas Bibliotecas Digitais UC Digitalis, UC Pombalina e UC Impactum, pressupõem a aceitação plena e sem reservas dos Termos e Condições de Uso destas Bibliotecas Digitais, disponíveis em https://digitalis.uc.pt/pt-pt/termos.

Conforme exposto nos referidos Termos e Condições de Uso, o descarregamento de títulos de acesso restrito requer uma licença válida de autorização devendo o utilizador aceder ao(s) documento(s) a partir de um endereço de IP da instituição detentora da supramencionada licença.

Ao utilizador é apenas permitido o descarregamento para uso pessoal, pelo que o emprego do(s) título(s) descarregado(s) para outro fim, designadamente comercial, carece de autorização do respetivo autor ou editor da obra.

Na medida em que todas as obras da UC Digitalis se encontram protegidas pelo Código do Direito de Autor e Direitos Conexos e demais legislação aplicável, toda a cópia, parcial ou total, deste documento, nos casos em que é legalmente admitida, deverá conter ou fazer-se acompanhar por este aviso.

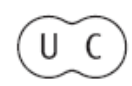




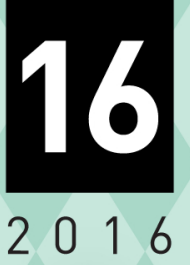

\section{Revista \\ de História \\ da Sociedade \\ e da \\ Cultura}




\title{
Paulmier de Gonneville et le Portugal: un navigateur normand dans la première mondialisation
}

\section{Paulmier de Gonneville and Portugal: a Norman navigator in the first globalization}

\author{
Christophe Maneuvrier \\ Université de Caen Normandie - CRAHAM/MRS \\ christophe.maneuvrier@unicaen.fr
}

Texto recebido em / Text submitted on: 24.02.2016

Texto aprovado em / Text approved on: 29.07.2016

Résumé: Connu partiellement depuis le XVII siècle et publié dans son intégralité dès 1869 , le récit de l'expédition faite par Binot Paulmier de Gonneville vers les Indes en 1503 et qui le conduisit au Brésil a fait l'objet depuis une vingtaine d'années d'une série de critiques qui, bien qu'infondées, ont jeté le discrédit sur son authenticité. Trop souvent étudié jusquà présent dans le cadre étroit des historiographies nationales (qu'elles soient française, portugaise ou brésilienne), le texte révèle, lorsqu'on l'intègre dans une lecture plus globale de l'histoire des échanges atlantiques, les liens entre les marins portugais et français. Entre navires européens, la concurrence peut être rude mais on se connaît, on se débauche, on échange des informations, on s'espionne aussi car les investisseurs français (ou plutôt normands) espèrent bien pouvoir eux aussi profiter de l'ouverture de la route vers les Indes orientales.

Mots-clés: Normandie, histoire globale, Brésil, Portugal, commerce atlantique.

Abstract: Partially known since the $17^{\text {th }}$ century and published in its entirety in 1869 , the account of the expedition made to India in 1503 by Binot Paulmier Gonneville that took him to Brazil has been the object of several criticisms that, although unfounded, have discredited its authenticity for around twenty years. So far too often studied in the narrow frontiers of national historiography (whether French, Portuguese or Brazilian), the text, when integrated into a more global understanding of the Atlantic trade's history, reveals links between Portuguese and French sailors. Competition could be fierce between European vessels, but they know each other, lay off, exchange information, and also spy each other, for the French (or rather Norman) investors hope to take advantage of the road to the East Indies opening.

Keywords: Normandy, global history, Brazil, Portugal, Atlantic trade. 


\section{Introduction}

Parti d'Honfleur le 24 juin 1503, à bord d'un navire de 120 tonneaux nommé L'Espoir, Paulmier de Gonneville fit d'abord route vers le sud atteignant les Canaries puis le Cap-Vert. De là, son navire obliqua vers l'ouest pour trouver les vents qui devaient le conduire jusqu'au sud du Cap de Bonne-Espérance mais il se perdit en mer avant d'accoster, le 6 janvier 1504, sur une terre inconnue de lui, l'actuel Brésil. Après 6 mois passés parmi les Amérindiens, il reprit la mer en compagnie de deux Amérindiens, dont l'un, Essomericq, était le fils du roi Arosca que Gonneville s'était engagé à ramener à son père dans les 20 mois suivants. Cependant, après une traversée épuisante, son navire fut piraté non loin de Guernesey par des pirates anglais et bretons. Paulmier de Gonneville, Essomericq, et quelques autres, réussirent à échapper aux forbans puis à regagner Honfleur, mais Gonneville ne put jamais retourner au Brésil et Essoméric passa le reste de sa vie en Normandie, adopté par le capitaine honfleurais. Connu par deux copies du milieu XVII ${ }^{e}$ siècle qui ne diffèrent l'une de l'autre que par certaines graphies, le texte qui relate ce voyage fut publié pour la première fois en intégralité par Marie-Armand d'Avezac, en $1869^{1}$. Depuis, il a été plusieurs fois réédité et a fait l'objet d'innombrables commentaires à tel point que l'on peut dire avec Ana Maria Pereira Ferreira que "praticamente todos os livros que tratam da Expansão Francesa falam nesta personagem e no seu relato” (Ferreira 1995: 143, n. 97).

Ce texte apparaît singulier à bien des égards. Considéré comme un récit de voyage - nous verrons plus loin que cette définition ne le caractérise que partiellement -, il fait figure d'unicum dans la production littéraire française du début du XVI ${ }^{\mathrm{e}}$ siècle et son authenticité a fait l'objet de nombreuses attaques depuis une vingtaine d'années. Familier depuis plus de trente ans des sources normandes et n'ayant aucun doute sur l'authenticité de ce texte, j'essaierai d'abord de montrer que la mauvaise compréhension de ce récit tient en grande partie à une insuffisante prise en compte des conditions dans lesquelles il fut rédigé. Dans un deuxième temps, je montrerai que, si le texte peut être considéré comme extraordinaire, le voyage de Gonneville l'est moins lorsqu'on tente de dépasser les lectures effectuées dans le cadre

\footnotetext{
D’Avezac 1869. D’Avezac ne connaissait que la copie conservée à la bibliothèque de l'Arsenal qui servit également aux éditions de Julien 1946: 25-49 et de Leyla Perrone-Moisés 1995. Une autre copie de 1659, conservée à la $\mathrm{BnF}$ (Fr. 7454, fls. 109-114), a été éditée par Pontharouart 2000. Cette dernière me semble être plus proche du texte original que celle publiée par D’Avezac. Une autre version du récit, plus courte, a été publiée par Pierre Margry 1867: 160-201 à partir d'une copie de 1783.
} 
d'une histoire nationale - qu'elle soit française, portugaise ou brésilienne - et lorsque l'on le replace dans un cadre plus global, celui de la première mondialisation. Avant-port de Rouen, Honfleur est en effet, jusquà la fondation du Havre en 1517, un port de niveau européen peuplé de marins qui sillonnent les mers depuis Hambourg jusqu'aux côtes de la Guinée ${ }^{2}$. Dans ce vaste espace, les marins français et portugais se connaissent, se fréquentent, et partagent une même culture maritime, au-delà des secrets mal préservés et des opérations d'espionnages mutuelles.

\section{Récit authentique ou forgerie du XVII ${ }^{\mathrm{e}}$ siècle?}

Le texte est perçu comme si singulier qu'il a récemment été considéré comme un faux forgé de toutes pièces au milieu du $\mathrm{XVI}^{\mathrm{e}}$ siècle. Ce mouvement hypercritique est né à partir de deux auteurs, l'un français, l'autre australienne. En France, la polémique a éclaté 1993 à l'initiative d'un auteur autodidacte, Jacques Lévêque de Pontharouart, qui affirma d'abord dans un article de presse puis dans un ouvrage publié en 2000 que le récit de Paulmier de Gonneville n'était qu'un faux "commis en 1659 par un abbé Paulmier de Courtonne, chanoine de Lisieux, qui prétendait être l'arrière-petit-fils du prince Essomericq" (Lévêque de Pontharouart 2000: 9). Largumentation repose principalement sur le fait que la terre décrite ne peut pas être le Brésil mais une "terre australe" qui n'existe pas. L'auteur affirme également que le texte ne respecte pas "les formes immuables du $16^{\mathrm{e}}$ siècle" (Lévêque de Pontharouart 2000: 24) et qu'un certain nombre de mots ne peuvent pas avoir été utilisés au $\mathrm{XVI}^{\mathrm{e}}$ siècle ${ }^{3}$. Malgré les contradictions apportées par plusieurs historiens de renom, ce scepticisme a connu depuis un assez large écho dans les média, notamment en Normandie où Paulmier de Gonneville était jusqualors perçu comme une sorte de héros régional.

Lorsqu'il publia son ouvrage, Jacques Lévêque de Pontharouart ignorait les travaux de Margaret Sankey, professeure de French studies à l'université de Sydney, spécialiste des premiers voyages français vers l’Australie, laquelle

\footnotetext{
La Relation du voyage de Gonneville indique que le navire n'avait auparavant "servi quà faire un seul voyage en Hambourg” (Perrone-Moisés 1995: 13). Sur la connaissance des côtes africaines, voir plus loin.

3 Le mot "barge" par exemple est présent dans le texte du Canarien. De même "médicament" est attesté dès 1314 (http://www.cnrtl.fr/etymologie/médicament, consulté en 2016.07.01); "Havre" dès 1135 chez Benoit de Sainte-Maure, "voyage” dès le XII siècle (http://www.cnrtl.fr/etymologie/ Havre, consulté en 2016.07.01).
} 
affirme depuis 1991 que le récit de Gonneville n'est qu'une forgerie du XVII siècle qualifiée même de fiction et d'utopie (Sankey 1991, 2013). En France, ses travaux furent surtout diffusés lors d'une journée d'études intitulée "Le Continent austral et le capitaine de Gonneville: vraie ou fausse relation de voyage?” organisée par François Moureau et Margaret Sankey à Tours le 14 mai 2007 et dont les contributions ont été publiées en 2013 dans le Australian Journal of French Studies (Sankey: 2013).

Il n'est pas question ici de répondre à toutes ces affirmations qui ont déjà fait l'objet de réponses puisqu'aucune n'est convaincante car, comme l'a écrit Geneviève Bouchon, "rien n'est plus facile que d'être négatif sous prétexte de scepticisme, et de rejeter ce qui dérange certaines traditions historiques" (Bouchon 1980: 235). Aucun historien sérieux connaissant bien le dossier n'accepte aujourd'hui l'idée qu'il s'agit d'une forgerie du XVII ${ }^{e}$ siècle. Ainsi, en 1993, Michel Mollat, Jean-Pierre Chaline et Philippe Bonnichon ont apporté une première réponse à Jacques de Pontharouart ${ }^{4}$. Par la suite, dans deux articles richement argumentés, Leyla Perrone-Moisés a répondu point par point aux affirmations de Jacques Lévêque de Pontharouart et de Margaret Sankey (Perrone-Moisés 2012, 2003). Le problème est cependant que les affirmations délirantes de ces auteurs ont créé la confusion chez les non-spécialistes francophones et même lusophones, comme Fernando Lourenço Fernandes qui, en 2009, a repris ces thèses dans un article paru dans la Revista do Instituto Histórico e Geográfico Brasileiro 5 . Ajoutons simplement au débat qu'il est absolument évident que le chanoine Jean Paulmier de Courtonne na pu inventer ce texte rédigé dans une langue qui est tout à fait conforme à celle des premières années du $\mathrm{XVI}^{e}$ siècle comme l'a bien montré René Lepelley, grand spécialiste de la langue normande (Lepelley 1993). De plus, les notes portées en marge de la copie conservée à la BnF par le chanoine Paulmier de Courtonne ne sont que des traductions en français de XVII siècle de termes et d'expressions qu'il ne comprenait pas et pour lesquels il eut besoin d'éclaircissement. Ces notes n'ont aucune raison d'être et deviennent simplement incompréhensibles si on voit en lui l'auteur de la Relation (Lévêque de Pontharouart 2000: 111-119).

\footnotetext{
4 Jean-Pierre Chaline a répondu en 1993 dans une tribune parue dans le quotidien rouennais Paris-Normandie du 18 mai 1993. La lettre de Philippe Bonnichon à Jacques Lévêque du 16/7/2001 n'est pas publiée mais elle est citée par Leblond 2013.

5 Fernandes 2009: 39-67. Parmi les francophones, on peut citer l'exemple de Monique Chastanet qui a malheureusement repris à son compte ces affirmations infondées (Chastanet 2010: 149-187).
} 


\section{Les limites des approches menées dans le cadre d'une histoire nationale}

Le chanoine Jean Paulmier de Courtonne, apparenté à Binot Paulmier de Gonneville, fut le premier, en 1663, à publier des extraits de la relation du voyage de son ancêtre dans ses Mémoires touchant l'établissement d'une mission chrestienne dans le Troisième Monde. Jusqu'au début du XIX ${ }^{\mathrm{e}}$ siècle, les auteurs pensèrent que la terre touchée par Gonneville était une "Terre Australe", localisée dans l'Océan Indien, Madagascar ou Australie. Depuis Pierre Margry, on sait qu'il s'agissait en réalité du Brésil, identification incontestée jusquà la sortie des ouvrages de Jacques Lévêque de Pontharouart et de Margaret Sankey ${ }^{6}$. Par la suite, et surtout à partir de la publication intégrale de 1869, la Relation a donné lieu à d'innombrables commentaires de la part d'historiens. Globalement, cette expédition - comme l'ensemble des installations françaises au Brésil effectuée durant le $\mathrm{XVI}^{\mathrm{e}}$ siècle - est perçue comme un échec démontrant l'impossibilité pour les marchands français de rivaliser avec les puissances espagnole et portugaise (Roncière 1906: 132-137) 7 .

Laffirmation selon laquelle le voyage de Binot Paulmier de Gonneville est une expédition isolée qui ne fut ni précédée ni suivie d’aucune autre ne relève pourtant que de l'utilisation de l'argument a silentio. Les procédés mis en œuvre par Gonneville sont d'ailleurs identiques à ceux auquel avaient recours les marins normands qui partaient vers Terre-Neuve et qui n'ont pourtant laissé aucun récit de leurs voyages: en 1509, sept hommes furent amenés à Rouen pour témoigner de la réussite d'une expédition vers Terre-Neuve et deux ans plus tard un homme originaire des mêmes contrées fut baptisé à Tréguier (Masse 2011: 83-106). Cette manière d'apporter une preuve à la revendication d'une découverte n'est d'ailleurs pas spécifique aux marins français puisque, dès 1501, Gaspar Corte Real avait ramené de Terre-Neuve à Lisbonne une cinquantaine de captifs (Masse 2011: 85).

Bien que traduite en portugais dès 1886 (Alencar Araripe 1886: 315360), la relation du voyage de Binot Paulmier de Gonneville est restée longtemps peu connue au Brésil (Verlinden 1959: 3-17), du moins jusqu’à sa réédition en 1992 par Leyla Perrone-Moisés (Perrone-Moisés 1992 et 1995). Avant cette date, les principaux travaux publiés au Brésil avaient été rédigés en portugais par des historiens francophones comme Michel Mollat ou Charles Verlinden (Verlinden 1959: 3-17; Mollat 1967). Pour les historiens

\footnotetext{
Pierre Margry consacre un long chapitre à "La navigation du capitaine de Gonneville et les prétentions des Normands à la découverte des terres australes sous Louis XII” (Margry 1867: 141-180).

Pour une approche similaire plus récente, voir par exemple Mérian 1993: 143-153.
} 
brésiliens, la Relation est perçue comme une source fondamentale pour l'histoire des sociétés amérindiennes à côté de documents portugais beaucoup plus célèbres, comme la lettre de Pêro Vaz de Caminha, inscrite depuis 2013 au registre Mémoire du Monde par l'UNESCO (Perrone-Moisés 1992: 116$130)^{8}$. Laurent Vidal a par ailleurs souligné que l'historiographie brésilienne (mais aussi française) voit dans la présence française au Brésil au XVI siècle une occasion perdue de mettre en place une autre colonisation, moins rude que la colonisation portugaise (Vidal 2000).

Les historiens portugais n'ont bien sûr pas ignoré la relation du voyage de Binot Paulmier de Gonneville, mais ils ne lui ont accordé qu'un intérêt limité, préférant consacrer leurs travaux sur les expéditions portugaises. Ainsi, dans son ouvrage de 1953, Luís de Matos lui consacre moins de deux pages pour ne retenir que le rôle des deux marins portugais qu'il est le premier à identifier (Matos 1952: 18-19). Plus récemment, Ana Maria Pereira Ferreira a vu en Gonneville un "pionnier" et un "prototype" - du moins pour la France - voire un "personnage de roman" et retient surtout le fait qu'il mène son expédition en compagnie de deux marins portugais".

Si le texte de la Relation a parfois été mal compris c'est parce que l'on s'est insuffisamment interrogé sur les raisons de sa production qui sont bien particulières puisqu'il s'agit d'une "Déclaration ... baillée vers justice par il capitaine et ses dits compagnons jouste qu'on requis les gens du roy nostre sire". La version courte précise quant à elle que les marins furent "semonchés par Monsieur le procureur du roy de faire notre déclaration de viage à notre retour sur la plainte de piratement de la dite navire" (Margry 1867: 160). C'est paradoxalement parce que l'opération fut un échec qu'elle a dû fournir ce récit à la justice royale: ayant été piraté par "un forban anglais dit Edouard Blunth de Plymouth" et plus encore par un "forban épineux, français de nation, savoir le capitaine Mouris Fortin, Breton, déjà condamné pour piraterie", Binot Paulmier de Gonneville était en droit de porter plainte devant les juridictions royales, mais pour que sa plainte ait quelque chance d'être reçue par l'administration et surtout d'aboutir, il lui fallait en faire un récit circonstancié. Sans doute la plainte visait-elle moins le forban anglais que Maurice

8 Cette approche n'est pas uniquement brésilienne, on la rencontre chez d'autres auteurs sud-américains dont certains hispanisants comme Miguel 2001: 177-181. La lettre de Pêro Vaz de Caminha été éditée à de nombreuses reprises. On peut en trouver une traduction française dans Perrone-Moisés 1992: 163-181.

9 "O protótipo encontra-se, e não é por acaso, na personagem quase de romance, do capitão de Gonneville, pioneiro, à falta de outros testemunhos, entre 1503-1505, do interesse da França pelo Novo Mundo" (Ferreira 1995: 143). 
Fortin, dont Gonneville prend bien soin de préciser qu'il fut auparavant déjà condamné pour le même crime.

\section{Le voyage de Gonneville dans le contexte de la première mondialisation}

Ces éléments nous incitent à tenter de replacer le voyage de Binot Paumier de Gonneville dans un mouvement qui dépasse le cadre national et à l'intégrer, comme nous y invite Sanjay Subrahmanyam, dans un mouvement beaucoup plus global (Subrahmanyam 2014: 199-200).

La Relation précise en effet que l'idée du voyage aux Indes vint à l'esprit de Paulmier de Gonneville à l’occasion d'un séjour à Lisbonne:

Et premièrement, disent que trafiquant en Lisbonne, il Gonneville et honorables hommes Jean l'Anglois et Pierre le Carpentier, vues les belles richesses d'épiceries et autres raretés venant en icelle cité de par les navires portugalloises allant ès Indes orientales depuis aucunes années découvertes, firent complot ensemble d'y envoyer une navire après bonne enquête à aucuns qui avaient fait tel voyage et pris à gros gages deux Portugallois qui en étaient revenus, l'un nommé Bastiam Moura l'autre Diègue Cohinto, pour en la route ès Indes aider de leur savoir (Relation ...).

Le texte contient simplement l'indication que les marins de Honfleur étaient "trafiquants en Lisbonne". On comprend que cela n'a rien d'exceptionnel, ni pour eux ni pour l'ensemble des marins de Normandie. Là, ils ont vu des navires revenir chargés d'épices et "autres raretés" rapportées des Indes orientales "découvertes" depuis plusieurs années. Ce qualificatif renvoie directement au vocabulaire utilisé par le roi du Portugal qui, dans sa lettre aux souverains de Castille, affirme avoir envoyé Vasco de Gama "à découvrir" (a descobrir), preuve que Paulmier de Gonneville sait parfaitement ce qui est en train de se passer à Lisbonne (Subrahmanyam 2014: 209).

Peut-on tenter de savoir quelle expédition portugaise Paulmier de Gonneville a vu revenir à Lisbonne? Il ne peut s'agir de la première expédition de Vasco de Gama puisqu'il est dit que ces voyages remontent alors à plusieurs années. Comme le navire dirigé par Gonneville partit le jour de la saint Jean-Baptiste 1503, cela ne peut pas être non plus le second voyage de Vasco de Gama qui, parti au début de l'année 1502, n’était pas encore rentré au moment où Paulmier de Gonneville quittait Honfleur. Restent la deuxième expédition lancée vers les Indes sous la conduite de Pedro Álvares Cabral et la troisième, celle qui fut placée sous le commandement de João da 
Nova. La première rentra au Portugal durant l'été 1501, entre le 23 juin pour la caravelle de Nicolau Coelho et le 21 juillet 1501 pour les autres navires. Or, on sait que le bilan de cette expédition fut mitigé puisque deux navires rentrèrent vides et plusieurs furent perdus, l'escadre revenant diminuée de moitié tandis que le bombardement de Calicut entraînait une rupture des relations commerciales avec cette ville. Les critiques envers cette expédition furent telles que le souverain se demanda même s'il fallait ou non continuer l'expérience (Subrahmanyam 2014: 230-236).

Il est donc plus probable que Gonneville ait été présent lors du retour de l'expédition de João da Nova, la plus mal connue des premières expéditions portugaises puisque tous les documents ou presque la concernant ont disparu (Bouchon 1980: 240). On sait cependant que, parti avec quatre caravelles pour Calicut en mars 1501, João da Nova était de retour à Lisbonne le 12 mars 1502 (Bouchon 1980: 252). Ce retour souleva l'enthousiasme même si les cargaisons étaient modestes et, comme pour les précédentes expéditions portugaises, une grande publicité entoura l'événement (Subrahmanyam 2014: 193-209). À cette date, les Vénitiens commençaient à regarder les expéditions portugaises d'un mauvais œil tant elles mettaient en péril leur maîtrise du commerce des épices à travers la Méditerranée d'autant que, comme l'écrit Sanjay Subrahmanyam, "il semblerait quà l'époque de cette expédition, la quatrième par la route du Cap, l'aventure se soit banalisée” (Subrahmanyam 2014: 243).

Impressionné par les marchandises déversées dans le port de Lisbonne, Paulmier de Gonneville ne se contente pas d'être un spectateur inactif. Il interroge de nombreux marins revenus des Indes ("après bonne enquête à aucuns qui avaient fait tel voyage") et prend "à gros gages deux Portugallois ... l'un nommé Bastiam Moura l'autre Diègue Cohinto, pour en la route ès Indes aider de leur savoir". Luís de Matos a corrigé les noms de ces marins en Sebastião de Moura et Diego Coutinho (Matos 1952: 19), mais on ne sait rien de ces personnages sinon qu'ils rentrèrent sains et sauf à Honfleur. Même leurs fonctions sur le navire ne sont pas précisées dans la Relation. On a parfois vu en eux des sortes de co-pilotes, mais ceci n'est qu'une extrapolation du texte car ils semblent plutôt avoir été de simples marins ne jouant qu'un rôle secondaire dans l'expédition. Leurs connaissances maritimes paraissent même avoir été limitées au point qu'ils se trompent lourdement lorsque, ayant vu des roseaux flotter sur la mer, ils se croient arrivés près du Cap de Bonne-Espérance alors qu'ils sont vraisemblablement non loin des îles Tristão da Cunha. La présence à bord de ces deux Portugais explique cependant la route suivie par Gonneville pour rejoindre les Indes orientales, 
seul objectif du Honfleurais puisque cette route, avec son escale prévue au Brésil, non loin du Cap Saint-Augustin, est exactement celle suivie par João da Nova (Bouchon 1980: 252).

Leur engagement à bord montre cependant que l'on est très facilement capable, de part et d'autre, de surmonter la barrière linguistique. Chacun observe l'autre, on discute, on "enquête", on s'espionne, aussi. Quel rôle jouent ces deux Portugais? On a pu les voir comme des traîtres à la cause du Portugal, mais ils peuvent tout aussi bien avoir été des espions au service de Manuel I ${ }^{\mathrm{er}}$ : au cas où Gonneville réussissait à atteindre les Indes orientales, il serait bien utile au roi du Portugal de connaître très exactement où il est allé et ce qu'il a pu y faire. Quelle connaissance Gonneville avait-il de Lisbonne? Était-ce son premier voyage ou bien connaissait-il la ville de longue date? Le fait que deux marins portugais acceptent de risquer leur vie avec lui nous laisse croire que le marin normand est connu à Lisbonne, au moins de réputation. Probablement n'en est-il pas à son premier séjour au Portugal.

Quel est le degré d'expérience et de connaissance de l'Atlantique de Gonneville lorsqu'il quitte Honfleur? Cette question ne peut trouver de réponse définitive, mais il est clair que Gonneville n'est pas un débutant et qu'il dispose d'un vaste réseau de relations au plus haut niveau de la marine française. Richard le Paulmier (vraisemblablement père ou oncle de Binot Paulmier de Gonneville) est signalé comme habitant de Honfleur vers 1460 puis comme "grenetier" du lieu, ce qui lui confère des fonctions d'ordre fiscal (Perret 1889: 100). En décembre 1487, il est fait procureur "conterolleur à Honnefleu et à la coste d'environ" par l'amiral de France Louis Malet de Graville. Le rôle de ce personnage dans l'expédition de 1503 n'est pas explicité par la Relation mais il est certain qu'elle se fit avec l'accord - sinon le soutien - de l'amiral de France dont le nom fut gravé, après celui du pape et celui du roi mais avant celui de Binot de Paulmier, sur la croix érigée au Brésil par l'équipage de L'Espoir (Perrone-Moisés 1995: 24). Immensément riche et proche du roi, Malet de Graville entretient un réseau considérable, au point d'être, en 1505, témoin du mariage entre Germaine de Foix et le roi Ferdinand II d'Aragon, veuf d'Isabelle la Catholique, et d'apposer sa marque au bas de leur contrat de mariage (Perret 1889: 191).

Avant-port maritime de Rouen, renforcé par le déclin d'Harfleur sur la rive droite de la Seine, Honfleur a bénéficié d'un développement considérable pendant la guerre de Cent Ans et ses capitaines sont des marins de premier plan (Pallu de la Barrière 2010 et 2012). Ainsi Guillaume Casenove, surnommé Coullon, "vielz admiral de la mer en Normandie" - ce qui en fait le lieutenant de Louis de Bourbon, amiral de France de 1461 à 1483 - s'est 
fait la spécialité de capturer les navires reliant Venise à la Flandre (Harrisse 1874: 10-13). En 1476, après la défaite de Toro, c'est lui qui est chargé d'escorter le roi Alfonse V vers la France. En septembre 1477, ce dernier passe un long moment à Honfleur, allant chaque matin se recueillir au sanctuaire des marins de Notre-Dame de Grâce, sur une colline dominant le port. C'est également à Honfleur qu'il rédige son testament avant d'y reprendre la mer pour rentrer au Portugal (Denis 1934: 317-318). En août 1485, Coullon le Jeune (neveu ou parent du précédent) et Georges Bissipat dit le Grec capturent quatre galères vénitiennes au large du Cap Saint-Vincent, provoquant un grave incident diplomatique entre la France et la République de Venise. Le roi eut beau ordonner la mise sous séquestre des biens volés, on ne put récupérer qu'un peu d'épices, 30 sacs de coton et 40 tonneaux de raisins de Corinthe alors que Charles VIII avait lui-même estimé les dommages subis par la République à au moins 200000 ducats. Venise envoie alors un ambassadeur à Honfleur pour tenter de régler l'affaire, mais une émeute éclate dans la ville lors de son arrivée et il est massacré par la foule dans la "Haulte-Rue", l'une des principales rues qui mènent au port. En réalité, les marins de Honfleur ne nient pas avoir caché le butin, d'autant qu'ils se savent soutenus par l'amiral Louis de Bourbon, lequel écrit au roi pour lui signifier qu'il est impossible de récupérer les biens des Vénitiens car "les mariniers en ont leurs pars et qu'ils ont vendues et transportées ainsy qu'ils ont voulu et sont les trois quarts desdits mariniers tous estrangers et sur lesquels il n'y a aucune rescousse", ajoutant même qu'il faut soutenir les marins sauf à prendre le risque de les voir partir s'installer en Angleterre (Perret 1889: 77). Le groupe des "mariniers" avec ses nombreux étrangers semble bien être un groupe international, et on comprend mieux à ces dires la facilité avec laquelle Gonneville put recruter les deux marins portugais.

Dans l'équipage de L'Espoir figurent deux "touristes", qui ont probablement acheté chèrement leur place et qui font confiance à son capitaine. L'un est "le sieur Coste, d'Harfleur, qui de curiosité venait au voyage", l'autre étant "Monsieur Nicole Le Febvre d'Honfleur qui était volontaire au voyage, curieux et personnage de savoir, ayant pourtrayé les façons; ce qui a été perdu avec les journaux de voyage lors du piratement de la navire" (Perrone-Moisés 1995: 21-24). On ignore qui était ce Nicole Le Febvre, capable de composer un étrange distique latin en forme de chronogramme. La présence d'un homme de culture dans cette expédition peut surprendre mais on sait qu'il existait à Honfleur une élite cultivée avide de connaissances sur le monde, comme en témoigne la présence à la même époque, chez Simon du Solier, d'un magnifique exemplaire de l'ouvrage de Marco Polo qui, provenant de 
la bibliothèque de Charles $\mathrm{V}$ puis de celle du Louvre où il se trouvait encore en 1424, est aujourd'hui conservé dans la bibliothèque royale de Suède (Delisle 1882: 423-424). Né vers 1451, qualifié en 1499 de procureur syndic des manants et habitants de Honfleur, Simon du Solier est alors tabellion (notaire) à Honfleur (Bréard et Barrey 1906: 249). Par ses fonctions, il connait nécessairement les armateurs et les capitaines de navires qui sont ses clients.

Les marins honfleurais n'ont pas qu'une connaissance livresque des contrées lointaines puisqu'ils sillonnent déjà les côtes de l'Afrique depuis plusieurs décennies. Les témoignages de ces expéditions maritimes sont rares et peu documentés, à l'image de celle commandée par Louis XI en 1483 qui, ayant appris qu'un habitant de Honfleur s'était procuré dans une île du Cap-Vert des remèdes pour la lèpre dont il était atteint, chargea Georges Bissipat dit le Grec, alors Capitaine de Honfleur, d'armer deux navires et une barque pour aller chercher ces remèdes au Cap-Vert. Afin de financer cette expédition, le roi imposa aux villes de Normandie un impôt exceptionnel de 30 à 40000 livres (Samaran 1924: 302-309). En raison du coût de l'opération et de l'importance de la flotte mise en œuvre, on peut légitimement s'interroger sur la vraie nature de cette opération. Avait-on réellement besoin de monter une expédition d'une telle importance pour aller seulement chercher quelques tortues ${ }^{10}$ ? Quoi qu'il en soit, on ne peut que constater que les marins de Honfleur fréquentaient assez facilement les îles du Cap-Vert et que ceci n’apparaissait alors pas exceptionnel.

Sans remonter à l'époque de Jean de Béthencourt qui, dès 1402, voulait ouvrir la route du fleuve de l'or (Maneuvrier 2016), on trouve dans le récit d'Eustache de la Fosse un détail qui montre bien que des marchands rouennais fréquentaient, dès le $\mathrm{XV}^{\mathrm{e}}$ siècle, les régions de l'actuel Sénégal. Eustache de la Fosse rapporte en effet qu'un certain Henry, facteur de Thomas Perrot, se perdit avec son navire à l'entrée du fleuve de l'or entraînant ainsi la banqueroute de son maitre Thomas Perrot, marchand de Bruges (Escudier 1992). Or, on sait que ce Thomas Perrot était le fils de Richard, qu'il était né à Rouen, et qu'il avait acquis la bourgeoisie de Bruges, le 27 juillet 1457 (Colens 1891: 327). Il y fit une belle carrière marchande réussissant même à se faire construire, en 1491, une chapelle dans la cathédrale de Bruges où il se fit inhumer en 1499. On y voyait autrefois son épitaphe ainsi que celle de son épouse, Anna De Wan, et celle de sa fille Anna (Gaillard 1861). Celle-ci

10 C'est Eustache de la Fosse qui rapporte dans son ouvrage la croyance selon laquelle la consommation de certaines tortues vivant dans les îles du Cap-Vert pouvait guérir de la lèpre; cf. Escudier 1992. 
indique qu'il était "haut Bourguignon" mais ses armes, de gueule à l'agnel d'argent et à trois étoiles à six branches, sont quasiment copiées sur celles de la ville de Rouen ${ }^{11}$.

\section{Conclusion}

Le récit du voyage de Paulmier est une source exceptionnelle pour l'histoire de l'Atlantique et de la première mondialisation et aucun élément sérieux ne permet de remettre en question son authenticité. Car la Relation du voyage de Gonneville ne doit pas être prise comme un récit destiné à conserver la mémoire des aventures de Gonneville mais bien comme une déposition faite devant une cour de justice pour faire aboutir une plainte contre des pirates. Sa singularité et son caractère exceptionnel ne doivent pas nous laisser croire que ce fut le seul voyage mené par des Français à travers l'Atlantique et l'indication selon laquelle Gonneville et son équipage furent à un moment "plus éloignés de l'Afrique que du pays des Indes occidentales où dempuis aucunes années en ça les Dieppois et les Malouins et autres Normands et Bretons vont quérir du bois à teindre en rouge, coton, guenons et perroquets et autres denrées, et que le vent d'est (...) les y poussant, fut d'unanimité délibéré d'aller quérir ce pays afin estout de se charger des susdites marchandises pour rescaper les frais et voyage" ne peut plus être rejetée d'un revers de main (Perrone-Moisés 1995: 26). Il n'est bien sûr pas question de rouvrir un débat stérile pour tenter de savoir qui, des Portugais ou des Français, furent les premiers européens à entrer en contact avec les Amérindiens du Brésil. $\mathrm{Au}$ contraire, nous souhaitons insister sur la proximité qui existe alors entre les navires et sur le caractère international de certains équipages. Il y a bien sûr de la concurrence entre les navires et les expéditions, mais on sait aussi se parler, s'échanger des informations, voire des marins. Au final, la véritable différence entre les expéditions portugaises et celles parties de France, c'est que les premières sont initiées et encadrées par le pouvoir royal tandis que les autres, comme celle de Gonneville, sont des entreprises privées, financées par des capitaux privés. Probablement étaient-ce là leurs principales limites et leur plus grande faiblesse.

11 La famille Perrot semble s'être bien installée à Bruges puisque Coline, fille de Thomas Perrot, épousa en 1465 un certain Colard De la Bie, lui aussi échevin (Gailliard 1846: 268). Elle décéda en 1495 et fut inhumée dans l'église Notre-Dame de Bruges (Noortvelde 1773: 248). 


\section{Bibliographie}

ALENCAR ARARIPE, Tristão de (1896). "Primeiro navio francez no Brazil”, Revista Trimestral do Instituto Histórico, geographico e ethnographico do Brazil, 49/2, 315-360.

BEAUCOURT DE NOORTVELDE (1773). Description historique de l'église collégiale et paroissiale de Notre-Dame à Bruges. Bruges: J. de Busscher.

BOUCHON, Geneviève (1980). "À propos de l'inscription de Colombo (1501). Quelques observations sur le premier voyage de João da Nova dans l'Océan Indien”, Revista da Universidade de Coimbra, 28, 233-270.

BRÉARD, Charles et Barrey, Philippe (1906). Documents relatifs à la marine normande au $\mathrm{XV}^{\mathrm{e}}$ et XVI ${ }^{\mathrm{e}}$ siècle. Rouen, 1906.

CHASTANET, Monique (2010). “Couscous à la sahélienne’ (Sénégal, Mali, Mauritanie)”, in H. Franconie, M. Chastagnet et François Sigaut (éd.), Couscous, boulgour et polenta. Transformer et consommer les céréales dans le monde. Paris: Karthala, 149-187.

COLENS, Jules (1891). “L'église Saint-Jean à Bruges. Ses tombes polychromées”, Annales de la société d'émulation pour l'étude de l'histoire et des Antiquités de la Flandre, 5 série, 4/41.

D’AVEZAC, Marie-Armand (1869). Campagne du navire L'Espoir de Honfleur (15031505). Relation authentique du voyage du capitaine de Gonneville ès nouvelles terres des Indes publiée intégralement pour la première fois avec une introduction et des éclaircissements. Paris: Challamel.

DELISLE, Léopold (1882). "Le Livre de Marco Polo, fac-similé d'un manuscrit du XIV siècle conservé à la Bibliothèque royale de Stockholm, par Nordenskiöld”, Bibliothèque de l'École des Chartes, 43/1, 226-235.

DELISLE, Léopold (1882). “Le Marco Polo de Stockholm”, Bibliothèque de l'École des Chartes, 43/1, 423-424.

DENIS, Serge (1934). “Le voyage en France d'Alphonse V de Portugal”, Bulletin Hispanique, 36/3, 289-318.

ESCUDIER, Denis (1992). Voyage d'Eustache Delafosse sur la côte de Guinée, au Portugal et en Espagne (1479-1481). Paris: Éditions Chandeigne.

FERNANDES, Fernando Lourenço (2009). "Os navegadores franceses na costa brasileira. No rastro do L'Espoir”, Revista do Instituto Histórico e Geográfico Brasileiro, 170/444, 39-67.

FERREIRA, Ana Maria Pereira (1986). "Corso francês e o comércio entre Portugal e as Canárias no século XVI (1521-1537)”, in VII Coloquio de Historia Canario-americano. Tomo 1. Las Palmas: Cabildo de Gran Canaria, 78-97.

FERREIRA, Ana Maria Pereira (1995). “O Brasil e a França na primeira metade do século XVI: viagens e interesses”, Arquipélago. História, 2a série, 1/1, 123-130.

FERREIRA, Ana Maria Pereira (1995). Problemas marítimos entre Portugal e a França na primeira metade do século XVI. Redondo: Patrimonia. 
GAILLARD, Victor Louis Marie (1861). Inscriptions funéraires et monumentales de la Flandre occidentale avec des données historiques et généalogiques. Tome 1. Bruges.

GAILLIARD, Jan Jacob (1846). Recherches historiques sur la Chapelle du Saint-Sang à Bruges. Bruges.

HARRISSE, Henry (1874). Les Colombo de France et d'Italie fameux marins du XVe siècle. 1461-1492. Paris: Tross.

JULIEN, Charles-André (1946). Les Français en Amérique pendant la première moitié du XVI ${ }^{\mathrm{e}}$ siècle. Paris: PUF.

LEBLOND, Jean (2013). "L’abbé Paulmier descendant d'un étranger des Terres australes? Notes sur la généalogie de l'abbé, la taxation des étrangers et la datation de la relation de voyage de Gonneville de 1505”, Australian Journal of French Studies, 50/1, 35-49 (http://online.liverpooluniversitypress.co.u/doi/abs/10.3828/AJFS.2013.3, consulté en 2016.06.30).

LEÓN-PORTILLA, Miguel (2001). "La muy temprana aportación etnográfica de Paulmier de Gonneville en 1504”, Caravelle, 76-77: Hommage à Georges Baudot, 177-181.

LEPELLEY, René (1993). "Les dialectismes normands dans le rapport du capitaine Binot Paulmier de Gonneville, navigateur honfleurais, sur son expédition de 1503 aux Amériques”, in Les Normands et les Amériques. Cahiers havrais de recherche historique, 17-24.

LÉVÊQUE DE PONTHAROUART, Jacques (2000). Paulmier de Gonneville. Son voyage imaginaire. Beauval-en-Caux: ASI Éditions.

MANEUVRIER, Christophe (à paraitre). "L'intention de monseigneur de Bethencourt est d'ouvrir le chemin du fleuve de l'or. Autour du rêve africain de Jean de Béthencourt”, Histoire monde, jeux d'échelles et espaces connectés, $47^{\mathrm{e}}$ Congrès de la SHMESP.

MARGRY, Pierre (1867). Les navigations françaises et la révolution maritime du XIV ${ }^{\mathrm{e}}$ au $\mathrm{XVI}^{\mathrm{e}}$ siècle. Paris: Tross.

MASSE, Vincent (2001). "Les sept hommes sauvages de 1509: fortune éditoriale de la première séquelle imprimée des contacts franco-amérindiens”, in Éditer la Nouvelle-France. Laval: Presses de l'Université, 83-106.

MATOS, Luís de (1952). Les Portugais en France au XVI ${ }^{e}$ siècle: études et documents, Coimbra: BGUC.

MÉRIAN, Jean-Yves (1993). “1500-1750, Les dynamiques de la conquête, l'opposition Espagne-Portugal en Amérique du Sud et les échecs de la France”, Mare Liberum: Revista de História dos Oceanos, 6, 143-153.

MOLLAT, Michel (1967). "As primeiras relações entre a França e o Brasil: dos Verrazani a Villegaignon", Revista de História, 70, 343-358 (http://revhistoria.usp.br/images/ stories/revistas/070/A003N070.pdf, consulté en 2016.06.30).

PALLU DE LA BARRIÈRE, Nathalie (2010). "Entre carrière militaire, commerce maritime et guerre de course: richesse et distinction à Honfleur (XIV $-X^{e}$ siècle)”, dans Laurence Jean-Marie et Christophe Maneuvrier (dir.) Distinction et supériorité sociale (Moyen Âge et époque moderne). Caen: Publications du CRAHM, 251-264. 
PALLU DE LA BARRIÈRE, Nathalie (2012). Honfleur et son arrière-pays, chronique d'un espace militaire (1367-1530). Paris. 3 vols. (thèse de doctorat policopiée).

PERRET, Paul-Michel (1889). Notice biographique sur Louis Malet de Graville: amiral de France (144?-1516). Paris: A. Picard.

PERRONE-MOISÉS, Leyla (1992). "Caminha e Gonneville: primeiros olhares sobre o Brasil”, Revista de História, 12. 116-130 (http://www.revistas.usp.br/revusp/article/ view/25582, consulté en 2016.06.30).

PERRONE-MOISÉS, Leyla (1992). Vinte Luas. Viagem de Paulmier de Gonneville ao Brasil: 1503-1505. São Paulo: Companhia das Letras.

PERRONE-MOISÉS, Leyla (1995). Le Voyage de Gonneville (1503-1505) et la découverte de la Normandie par les Indiens du Brésil. Étude et commentaire. Paris: Éditions Chandeigne.

PERRONE-MOISÉS, Leyla (2003). “Le voyage de Gonneville a-t-il vraiment eu lieu?”, in Colloque International "Voyageurs et images du Brésil" MSH-Paris, le 10 décembre 2003. Table 2: Les récits de conquête et de colonisation (http://editions-villegagnons. com/GONNEVILLE.pdf, consulté en 2016.06.30).

PERRONE-MOISÉS, Leyla (2012). “Le voyage de Gonneville: un défi à l'historiographie”, in Actes du colloque Rouen 1562. Montaigne et les Cannibales, Centre d'études et de recherche Éditer/Interpréter, octobre 2012 (http://ceredi.labos.univ-rouen.fr/public/?le-voyage-de-gonneville-un-defi-a.html, consulté en 2016.06.30).

RONCIÈRE, Charles de la (1906). Histoire de la marine française. Tome 3. Paris: Plon.

SAMARAN, Charles (1924). "Une page inédite de l'Histoire de Louis XI par Thomas Basin”, Bibliothèque de l'École des Chartes, 85, 302-309.

SANKEY, Margaret (1991). "La mission impossible: l'abbé Paulmier et les Terres australes", in Bruce Merry (éd.), Essays in Honour of Keith Val Sinclair. Townsville: James Cook University of North Queensland, 35-47.

SANKEY, Margaret (2013). "The abbé Jean Paulmier and French missions in the Terres australes: myth and history”, Australian Journal of French Studies, 50/1, 3-15 (http:// online.liverpooluniversitypress.co.uk/toc/ajfs/50/1, consulté en 2016.06.30).

SUBRAHMANYAM, Sanjay (2014). Vasco de Gama: légende et tribulations du vice-roi des Indes. Paris: le Seuil.

VERLINDEN, Charles (1949). "Deux aspects de l'expansion commerciale du Portugal au Moyen Âge (Honfleur au XIV siècle. Middelbourg au XIV et au XVe)”, Revista Portuguesa de História, 4, 169-209.

VERLINDEN, Charles (1959). "Paulmier de Gonnevile e os índios do Brasil em 1544", Revista de História, 39, 3-17 (http://revhistoria.usp.br/images/stories/revistas/039/ A001N039.pdf, consulté en 2016.06.30).

VIDAL, Laurent (2000). "La présence française dans le Brésil au XVI ${ }^{e}$ siècle”, Cahiers des Amériques latines, 34, 17-38 (http://www.iheal.univ-paris3.fr/sites/www.iheal.univ-paris3.fr/files/Cal_034.pdf, consulté en 2016.06.30). 